\title{
Existence of Solutions for G-SFDEs with Cauchy-Maruyama Approximation Scheme
}

\author{
Faiz Faizullah \\ Department of Basic Sciences and Humanities, College of Electrical and Mechanical Engineering, National University of Sciences \\ and Technology (NUST), Pakistan
}

Correspondence should be addressed to Faiz Faizullah; faiz_math@ceme.nust.edu.pk

Received 31 May 2014; Revised 21 July 2014; Accepted 19 August 2014; Published 11 September 2014

Academic Editor: Saeed Islam

Copyright (c) 2014 Faiz Faizullah. This is an open access article distributed under the Creative Commons Attribution License, which permits unrestricted use, distribution, and reproduction in any medium, provided the original work is properly cited.

We present the Cauchy-Maruyama (CM) approximation scheme and establish the existence theory of stochastic functional differential equations driven by G-Brownian motion (G-SFDEs). Several useful properties of Cauchy-Maruyama (CM) approximate solutions $X^{k}$ of G-SFDEs are given. We show that the unique solution of G-SFDEs gets convergence from Cauchy-Maruyama (CM) approximate solutions. The existence theorem for G-SFDEs is developed with the above mentioned scheme.

\section{Introduction}

A significant role is played by stochastic differential equations (SDEs) in a broad range of applied disciplines, including biology, economics, finance, chemistry, physics, microelectronics, and mechanics. In many applications, one assumes that the future state of the system is independent of the past states. However, under close scrutiny, it becomes apparent that a more realistic model would contain some of the past state of the system and one needs stochastic functional differential equations to formulate such systems [1]. Also see $[2,3]$. The motion theory of G-Brown, corresponding to Itô's calculus and stochastic differential equations driven by G-Brownian motion (G-SDEs), was introduced by Peng $[4,5]$. Then many authors paid much attention to this theory and G-SDEs [6-10]. The existence theory for solutions of stochastic functional differential equations driven by GBrownian motion (G-SFDEs) was introduced by Ren et al. via Picard approximation [11]. However, like the classical stochastic differential equations, the convergence of Picard approximate solutions to the unique solution of G-SFDEs under the linear growth and Lipschitz condition is still open [1]. In contrast, here we introduce the Cauchy-Maruyama approximation scheme for G-SFDEs and show that the unique solution $X(t)$ of G-SFDEs gets convergence from CM approximate solutions $X^{k}(t), k \geq 1$. Furthermore, using CM approximation scheme, it is shown that the G-SFDE has a unique solution.

Some mathematical preliminaries are given in subsequent section. In Section 3, the CM approximation scheme for GSFDEs is developed. In Section 4, some properties of the CM approximate solutions are presented. In Section 5, the theory of existence for the solutions of G-SFDEs with the above stated method is established.

\section{Basic Notions}

Some basic definitions and notions are presented in this section $[4,5,11-13]$. Suppose that if $\Lambda$ is a basic space that is nonempty and $\mathscr{H}$ is a space of linear real valued mappings stated on the space $\Lambda$ such as $b \in \mathscr{H}$, where $b$ is any arbitrary constant and if $Y_{1}, Y_{2}, \ldots, Y_{n} \in \mathscr{H}, \varphi\left(Y_{1}, Y_{2}, \ldots, Y_{n}\right) \in \mathscr{H}$ for every $\varphi \in \mathbb{C}_{\text {l. Lip }}\left(\mathbb{R}^{n}\right)$. Furthermore,

$$
\begin{aligned}
& \mathbb{C}_{l . \text { Lip }}\left(\mathbb{R}^{n}\right) \\
& =\left\{\varphi: \mathbb{R}^{n} \longrightarrow \mathbb{R} \mid \exists C \in \mathbb{R}^{+},\right. \\
& q \in \mathbb{Z}^{+} \text {s.t. }|\varphi(y)-\varphi(z)| \\
& \left.\leq C\left(1+|y|^{q}+|z|^{q}\right)|y-z|\right\},
\end{aligned}
$$


for $y, z \in \mathbb{R}^{n}$; that is, $\mathbb{C}_{l \text {. Lip }}\left(\mathbb{R}^{n}\right)$ is a space containing the linear mappings $\varphi$. Here $\mathscr{H}$ represents the space of random variables.

Definition 1. A functional $\mathscr{E}: \mathscr{H} \rightarrow \mathbb{R}$ is named a sublinear expectation, if for every $Y, Z \in \mathscr{H}, \theta \geq 0$, and $b \in \mathbb{R}$ it holds the following monotonic, subadditivity, constant preserving and positive homogeneity properties, respectively:

(i) $Z \geq Y$ implies $\mathscr{E}[Z] \geq \mathscr{E}[Y]$.

(ii) $\mathscr{E}[Z+Y] \leq \mathscr{E}[Z]+\mathscr{E}[Y]$.

(iii) $\mathscr{E}[b]=b$.

(iv) $\mathscr{E}[\theta Y]=\theta \mathscr{E}[Y]$.

The space given by triple $(\Lambda, \mathscr{H}, \mathscr{E})$ is named sublinear expectation space. The functional $\mathscr{E}$ is known as a nonlinear expectation if it holds only the first two properties.

Definition 2. Assume two random vectors $Y$ and $Z$, which are $n$-dimensional and are, respectively, defined on the spaces $(\Lambda, \mathscr{H}, \mathscr{E})$ and $(\widetilde{\Lambda}, \widetilde{\mathscr{H}}, \widetilde{\mathscr{E}})$. These are distributed identically, represented as $Y \sim Z$ if

$$
\mathscr{E}[\varphi(Y)]=\widetilde{\mathscr{E}}[\varphi(Z)], \quad \forall \varphi \in \mathbb{C}_{l . \operatorname{Lip}}\left(\mathbb{R}^{n}\right) .
$$

Definition 3. Suppose $(\Lambda, \mathscr{H}, \mathscr{E})$ is a sublinear expectation space with $Y \in \mathscr{H}$ and

$$
\bar{\sigma}^{2}=\mathscr{E}\left[Y^{2}\right], \quad \underline{\sigma}^{2}=-\mathscr{E}\left[-Y^{2}\right]
$$

Then $Y$ is called $\mathcal{N}\left(0 ;\left[\bar{\sigma}^{2}, \underline{\sigma}^{2}\right]\right)$-distributed or G-distributed, if for every $c, d \geq 0$ we get

$$
c Y+d Z \sim \sqrt{c^{2}+d^{2}} Y
$$

for every $Z \in \mathscr{H}$, where $Z \sim Y$ and it is independent of $Y$

To define the G-Brownian motion, we suppose that $\Lambda=$ $C_{0}\left(\mathbb{R}^{+}\right)$represents the space of all real valued incessant paths $\left(w_{t}\right)_{t \in \mathbb{R}^{+}}$such that $w_{0}=0$ having norm

$$
\rho\left(w^{1}, w^{2}\right)=\sum_{k=1}^{\infty} \frac{1}{2^{k}}\left(\max _{t \in[0, k]}\left|w_{t}^{1}-w_{t}^{2}\right| \wedge 1\right) .
$$

Consider for $t \in[0, \infty)$ and $w \in \Lambda$ the process $B_{t}(w)=w_{t}$, which is commonly known as the canonical process. Then for every rigid $T \in[0, \infty)$ we get that

$$
\begin{aligned}
& L_{\text {ir }}\left(\Lambda_{T}\right) \\
& =\left\{\varphi\left(B_{t_{1}}, B_{t_{2}}, \ldots, B_{t_{n}}\right): t_{1}, \ldots, t_{n} \in[0, T],\right. \\
& \left.\quad n \in \mathbb{Z}^{+}, \varphi \in \mathbb{C}_{l . \text { Lip }}\left(\mathbb{R}^{n}\right)\right\},
\end{aligned}
$$

for $t \leq T, L_{i r}\left(\Lambda_{t}\right) \subseteq L_{i r}\left(\Lambda_{T}\right)$, and $L_{i r}(\Lambda)=\cup_{m=1}^{\infty} L_{i r}\left(\Lambda_{m}\right)$. Take $\left\{\xi_{k}\right\}_{k=1}^{\infty}$ which is a sequence of $n$-dimensional random vectors on the space $(\Lambda, \mathscr{H}, \mathscr{E})$. Also, for every $k=$ $1,2, \ldots, n-1, \xi_{k+1}$ is independent of $\left(\xi_{1}, \xi_{2}, \ldots, \xi_{k}\right)$ and $\xi_{k}$ is $\mathcal{N}\left(0 ;\left[\bar{\sigma}^{2}, \underline{\sigma}^{2}\right]\right)$-distributed. Then we represent a sublinear expectation $\mathscr{E}[\cdot]$ stated on $L_{i r}(\Lambda)$ as follows. For every $Y=$ $\varphi\left(B_{t_{1}}-B_{t_{0}}, B_{t_{2}}-B_{t_{1}}, \ldots, B_{t_{n}}-B_{t_{n-1}}\right) \in L_{\text {ir }}(\Lambda), 0=t_{0}<t_{1}<$ $\cdots<t_{n}<\infty$, and $\varphi \in \mathbb{C}_{l \text {. Lip }}\left(\mathbb{R}^{n}\right)$ we have

$$
\begin{aligned}
\mathscr{E} & {\left[\varphi\left(B_{t_{1}}-B_{t_{0}}, B_{t_{2}}-B_{t_{1}}, \ldots, B_{t_{n}}-B_{t_{n-1}}\right)\right] } \\
= & \widetilde{\mathscr{E}}\left[\varphi\left(\sqrt{t_{1}-t_{0}} \xi_{1}, \ldots, \sqrt{t_{n}-t_{n-1}} \xi_{n}\right)\right] .
\end{aligned}
$$

Definition 4. The expectation mentioned above, that is, $\mathscr{E}$ : $L_{i r}(\Lambda) \rightarrow \mathbb{R}$, is known as a G-expectation and the related process $\left\{B_{t}, t \geq 0\right\}$ is said to be a G-Brownian motion.

The completion of $L_{i r}(\Lambda)$ is presented with the norm $\|X\|_{r}=\left(\mathbb{E}\left[|X|^{r}\right]\right)^{1 / r}$ for $r \geq 1$ by $L_{G}^{r}(\Lambda)$. For $0 \leq t \leq T<\infty$ we have $L_{G}^{r}\left(\Lambda_{t}\right) \subseteq L_{G}^{r}\left(\Lambda_{T}\right) \subseteq L_{G}^{r}(\Lambda)$. Also, $\mathscr{F}_{t}=\sigma\left\{B_{s}, 0 \leq s \leq\right.$ $t\}, \mathscr{F}=\left\{\mathscr{F}_{t}\right\}_{t \geq 0}$, denotes the filtration caused due to the stated process $\left\{B_{t}, t \geq 0\right\}$. To define G-Itô's integral, we consider, for every $T \in \mathbb{R}^{+}$, a partition of $[0, T]$ which is a finite ordered subset $\pi_{T}=\left\{t_{0}, t_{1}, \ldots, t_{N}\right\}$ where $T=t_{N}>t_{N-1}>\cdots>t_{1}>$ $t_{0}=0$. Assume $r \geq 1$ to be fixed. For the above mentioned given partition $\pi_{T}$ we represent

$$
\begin{aligned}
& M_{G}^{r, 0}(0, T) \\
& =\left\{\eta_{t}(w)=\sum_{k=0}^{N-1} \xi_{k}(w) I_{\left[t_{k}, t_{k+1}\right]}(t): \xi_{k} \in L_{G}^{r}\left(\mathscr{F}_{t_{k}}\right),\right. \\
& \left.t_{k}<t_{k+1}, k=0,1, \ldots, N-1\right\} .
\end{aligned}
$$

Remember that $L_{G}^{r}\left(\mathscr{F}_{t}\right)=\left\{\xi \in L_{G}^{1}\left(\mathscr{F}_{t}\right) ; \mathbb{E}\left[|\xi|^{r}\right]<\infty\right\}$. We use $M_{G}^{r}(0, T)$ to denote the completion of $M_{G}^{r, 0}(0, T)$ under the norm $\|\eta\|=\left\{\int_{0}^{T} \mathscr{E}\left[\left|\eta_{v}\right|^{r}\right] d v\right\}^{1 / r}$ where for $1 \leq r \leq p$, $M_{G}^{r}(0, T) \supset M_{G}^{p}(0, T)$.

Definition 5. For every $\eta_{t} \in M_{G}^{2,0}(0, T)$, G-Itô's integral is denoted by $I\left(\eta_{t}\right)$ and is defined by

$$
I(\eta)=\sum_{k=0}^{N-1} \xi_{k}\left(B_{t_{k+1}}-B_{t_{k}}\right)=\int_{0}^{T} \eta_{v} d B_{v} .
$$

Let a sequence of partition of $[0, t]$ be given by $\pi_{T}^{N}=$ $\left\{t_{0}^{N}, t_{1}^{N}, \ldots, t_{N}^{N}\right\}, N=1,2,3, \ldots$, where $\pi_{t}^{N}=\max _{1 \leq k \leq N} \mid t_{k}^{N}-$ $t_{k-1}^{N} \mid$.

Definition 6. The process $\left\{\langle B\rangle_{t}, t \geq 0\right\}$, known as quadratic variation process of $\left\{B_{t}, t \geq 0\right\}$, is a continuous increasing process having $\langle B\rangle_{0}=0$ and is stated by

$$
\begin{aligned}
\langle B\rangle_{t} & =B_{t}^{2}-2 \int_{0}^{t} B_{v} d B_{v} \\
& =\lim _{\pi_{t}^{N} \rightarrow \infty} \sum_{k=0}^{N-1} \xi_{k}\left(B_{t_{k+1}}-B_{t_{k}}\right)^{2} .
\end{aligned}
$$

Definition 7. Suppose $\mathscr{B}(\Lambda)$ is the Borel $\sigma$-algebra of $\Lambda$ and $\mathscr{P}$ is the (weakly compact) combination of measures for 
probability $P$ that is stated on $(\Lambda, \mathscr{B}(\Lambda))$. Afterwards, the capacity $\widehat{c}(\cdot)$ attached to $\mathscr{P}$ is given by

$$
\widehat{c}(D)=\sup _{P \in \mathscr{P}} P(D), \quad D \in \mathscr{B}(\Lambda) .
$$

In case of the capacity of a set $D$ to be zero then the set $D$ is called polar, that is, $\widehat{c}(D)=0$ and a property carries quasisurely (in short q.s.) if it is satisfied outside a polar set.

Lemma 8 (Gronwall inequality). Let $\kappa$ and $c<d$ be real numbers, $g(t) \geq 0$ for $t \in[c, d]$, and $h(t)$ be a real valued continuous function on $[c, d]$ such that $h(t) \leq \kappa+$ $\int_{c}^{d} g(v) h(v) d v ;$ then

$$
h(t) \leq \kappa e^{\int_{c}^{d} g(v) d v}, \quad t \in[c, d] .
$$

\section{The Cauchy-Maruyama Approximation Scheme for G-SFDEs}

Let $0=t_{0} \leq t \leq T<\infty, a \in[-\tau, 0]$, and $\tau>0$. Represent by $B C\left([-\tau, 0] ; \mathbb{R}^{d}\right)$ the space of bounded incessant $\mathbb{R}^{d}$-valued mappings $\varphi$ defined on $[-\tau, 0]$ and having norm $\|\varphi\|=\sup _{a \leq 0}|\varphi(a)|$. Suppose $\alpha:\left[t_{0}, T\right] \times B C\left([-\tau, 0] ; \mathbb{R}^{d}\right) \rightarrow$ $\mathbb{R}^{d}, \beta:\left[t_{0}, T\right] \times B C\left([-\tau, 0] \mathbb{R}^{d}\right) \rightarrow \mathbb{R}^{d \times m}$, and $\gamma:\left[t_{0}, T\right] \times$ $B C\left([-\tau, 0] ; \mathbb{R}^{d}\right) \rightarrow \mathbb{R}^{d \times m}$ are Borel measurable. Assume the following $d$-dimensional G-SFDE [5]:

$$
\begin{aligned}
& d Y(t) \\
& =\alpha(t, Y(t+a)) d t+\beta(t, Y(t+a)) d\langle B, B\rangle(t) \\
& \quad+\gamma(t, Y(t+a)) d B(t), \quad t \in\left[t_{0}, T\right],
\end{aligned}
$$

with initial data

$$
\begin{gathered}
Y_{t_{0}}=\xi=\{\xi(a):-\tau \leq a \leq 0\} \text { is } \mathscr{F}_{0} \text {-measurable, } \\
B C\left([-\tau, 0] ; \mathbb{R}^{d}\right) \text {-value random variable }
\end{gathered}
$$

$$
\text { so that } \xi \in M_{G}^{2}\left([-\tau, T] ; \mathbb{R}^{d}\right) \text {. }
$$

Also, $\{\langle B, B\rangle(t): t \geq 0\}$ represent the process of quadratic variation of $\mathrm{G}$-Brownian motion $\{B(t): t \geq 0\}$. The coefficients $\alpha, \beta$, and $\gamma$ are given mappings satisfying $\alpha(\cdot, y)$, $\beta(\cdot, y), \gamma(\cdot, y) \in M_{G}^{2}\left(\left[t_{0}-\tau, T\right] ; \mathbb{R}^{d}\right)$ for all $y \in \mathbb{R}^{d}$. The integral form of (13) with initial data (14) is given as the following:

$$
\begin{gathered}
Y(t) \\
=\xi(0)+\int_{t_{0}}^{t} \alpha(v, Y(v+a)) d v \\
\quad+\int_{t_{0}}^{t} \beta(v, Y(v+a)) d\langle B, B\rangle(v) \\
+\int_{t_{0}}^{t} \gamma(v, Y(v+a)) d B(v), \\
\quad t \in\left[t_{0}, T\right],
\end{gathered}
$$

where $\xi(0) \in \mathbb{R}^{d}$ is a given initial condition.
Definition 9. An $\mathbb{R}^{d}$-valued stochastic process $Y(t)$ defined on $t_{0}-\tau \leq t \leq T$ is said to be the solution of (13) having initial data (14) if it holds the properties given below:

(i) the solution $Y(t)$ is continuous and it is $\mathscr{F}_{t}$-adapted, for all $0=t_{0} \leq t \leq T$;

(ii) $\alpha\left(t, Y_{t}\right) \in \mathscr{L}^{1}\left([0, T] ; \mathbb{R}^{d}\right)$ and $\beta\left(t, Y_{t}\right), \gamma\left(t, Y_{t}\right) \quad \epsilon$ $\mathscr{L}^{2}\left([0, T] ; \mathbb{R}^{d \times m}\right) ;$

(iii) $Y_{t_{0}}=\xi$ and for every $t \in\left[t_{0}, T\right]$

$Y(t)$

$$
\begin{gathered}
=\xi(0)+\int_{t_{0}}^{t} \alpha(v, Y(v+a)) d v \\
+\int_{t_{0}}^{t} \beta(v, Y(v+a)) d\langle B, B\rangle(v) \\
+\int_{t_{0}}^{t} \gamma(v, Y(v+a)) d B(v) \text { q.s. }
\end{gathered}
$$

The Cauchy-Maruyama approximation scheme for GSFDE (15) with initial data (14) is shown as the following. For any integer $k \geq 1$, we define $Y^{k}(t)$ on $\left[t_{0}-\tau, T\right]$ as follows:

$$
Y^{k}\left(t_{0}+a\right)=\xi(a) \quad \text { for }-\tau \leq a \leq 0,
$$

$Y^{k}(t)$

$$
\begin{aligned}
= & Y^{k}\left(t_{0}+\frac{n}{k}\right)+\int_{t_{0}+(n / k)}^{t} \alpha\left(v, Y^{k}\left(t_{0}+\frac{n}{k}+a\right)\right) d v \\
& +\int_{t_{0}+(n / k)}^{t} \beta\left(v, Y^{k}\left(t_{0}+\frac{n}{k}+a\right)\right) d\langle B, B\rangle(v) \\
& +\int_{t_{0}+(n / k)}^{t} \gamma\left(v, Y^{k}\left(t_{0}+\frac{n}{k}+a\right)\right) d B(v),
\end{aligned}
$$

for $t_{0}+(n / k)<t \leq\left(t_{0}+((n+1) / k)\right) \wedge T, n=$ $0,1,2, \ldots$ Obviously, $Y^{k}(\cdot)$ can be determined by stepwise iterations over the interval $\left(t_{0}, t_{0}+(1 / n)\right],\left(t_{0}+(1 / n), t_{0}+\right.$ $(2 / n)]$, and so forth. Furthermore, if we define $Y^{k}\left(t_{0}+a\right)=$ $\tilde{Y}^{k}\left(t_{0}\right)$ and $Y^{k}\left(t_{0}+(n / k)+a\right)=\widetilde{Y}^{k}(t)$ for $t_{0}+(n / k)<t \leq$ $\left(t_{0}+((n+1) / k)\right) \wedge T, n=0,1,2, \ldots$, then (18) yields

$$
\begin{aligned}
& Y^{k}(t) \\
& =\xi(0)+\int_{t_{0}}^{t} \alpha\left(v, \tilde{Y}^{k}(v) d v\right) \\
& \quad+\int_{t_{0}}^{t} \beta\left(v, \tilde{Y}^{k}(v)\right) d\langle B, B\rangle(v) \\
& \quad+\int_{t_{0}}^{t} \gamma\left(v, \tilde{Y}^{k}(v)\right) d B(v),
\end{aligned}
$$

for $t_{0} \leq t \leq T$. We will use the following linear growth 
and Lipschitz conditions, respectively. Suppose two positive numbers $\widehat{K}$ and $\widetilde{K}$ such that

(i) for every $t \in\left[t_{0}, T\right]$,

$$
|\alpha(t, z)|^{2}+|\beta(t, z)|^{2}+|\gamma(t, z)|^{2} \leq \widetilde{K}\left(1+|z|^{2}\right),
$$

(ii) for all $y, z \in\left(\left[t_{0}-\tau, T\right] R^{d}\right)$ and $t \in\left[t_{0}, T\right]$,

$$
\begin{gathered}
|\alpha(t, y)-\alpha(t, z)|^{2}+|\beta(t, y)-\beta(t, z)|^{2} \\
+|\gamma(t, y)-\gamma(t, z)|^{2} \leq \widehat{K}|y-z|^{2} .
\end{gathered}
$$

\section{Properties of Cauchy-Maruyama Approximate Solutions}

Here we show that the Cauchy-Maruyama approximate solutions $Y^{k}(t)$ satisfy some very useful properties, which are given in the form of Lemmas (10) and (12).

Lemma 10. Let the linear growth condition (20) hold. Then for all $k \geq 1$,

$$
\sup _{t_{0}-\tau \leq t \leq T} \mathscr{E}\left[\left|Y^{k}(t)\right|^{2}\right] \leq C, \quad \text { for any } T>0,
$$

where $C=\mathscr{E}\left[\|\xi\|^{2}\right]+K_{1} e^{4 \widetilde{K} K_{2} T}, K_{1}=4\left(1+T \widetilde{K} K_{2}\right) \mathscr{E}\left[\|\xi\|^{2}\right]+$ $4 \widetilde{K} K_{2} T, K_{2}=C_{1} T+C_{2} T+C_{3}$, and $C_{1}, C_{2}$, and $C_{3}$ are arbitrary positive constants.

Proof. Using the basic inequality $(a+b+c+d)^{2} \leq 4 a^{2}+4 b^{2}+$ $4 c^{2}+4 d^{2}$ then from (19) we have

$$
\begin{aligned}
& \left|X^{k}(t)\right|^{2} \\
& \leq 4|\xi(0)|^{2}+4\left|\int_{t_{0}}^{t} \alpha\left(v, \widetilde{Y}^{k}(v)\right) d v\right|^{2} \\
& \quad+4\left|\int_{t_{0}}^{t} \beta\left(v, \widetilde{Y}^{k}(v)\right) d\langle B, B\rangle(v)\right|^{2} \\
& \quad+4\left|\int_{t_{0}}^{t} \gamma\left(v, \widetilde{Y}^{k}(v)\right) d B(v)\right|^{2},
\end{aligned}
$$

for $t \in\left[t_{0}, T\right]$. Applying the Burkholder-Davis-Gundy (BDG) inequalities [9] and the linear growth condition (20) and taking sublinear expectation, it is implied that

$$
\begin{aligned}
\mathscr{E}\left[\sup _{t_{0} \leq u \leq t}\left|Y^{k}(u)\right|^{2}\right] \\
\leq 4 \mathscr{E}\left[\|\xi\|^{2}\right]+4 \widetilde{K}\left(C_{1} T+C_{2} T+C_{3}\right) \\
\quad \times \int_{t_{0}}^{t}\left(1+\mathscr{E}\left[\left|\widetilde{Y}^{k}(v)\right|^{2}\right]\right) d v \\
\leq 4 \mathscr{E}\left[\|\xi\|^{2}\right]+4 \widetilde{K}\left(C_{1} T+C_{2} T+C_{3}\right) T \\
\quad+4 \widetilde{K}\left(C_{1} T+C_{2} T+C_{3}\right) \\
\quad \times \int_{t_{0}}^{t} \mathscr{E}\left[\left|\widetilde{Y}^{k}(v)\right|^{2}\right] d v .
\end{aligned}
$$

Remembering the notion of $\widetilde{Y}^{k}(t)$, we proceed as

$$
\begin{aligned}
\mathscr{E}\left[\sup _{t_{0} \leq u \leq t}\left|Y^{k}(u)\right|^{2}\right] \\
\leq 4 \mathscr{E}\left[\|\xi\|^{2}\right]+4 \widetilde{K}\left(C_{1} T+C_{2} T+C_{3}\right) T \\
\quad+4 \widetilde{K}\left(C_{1} T+C_{2} T+C_{3}\right) \\
\quad \times \int_{t_{0}}^{t} \mathscr{E}\left[\sup _{t_{0}-\tau \leq u \leq v}\left|Y^{k}(u)\right|^{2}\right] d v .
\end{aligned}
$$

Noting the fact that

$$
\begin{aligned}
& \sup _{t_{0}-\tau \leq t \leq T}\left|Y^{k}(t)\right|^{2} \\
& \quad \leq\|\xi\|^{2}+\sup _{t_{0} \leq t \leq T}\left|Y^{k}(t)\right|^{2}, \\
& \mathscr{E}\left[\sup _{t_{0}-\tau \leq t \leq T}\left|Y^{k}(t)\right|^{2}\right] \\
& \quad \leq \mathscr{E}\left[\|\xi\|^{2}\right]+\mathscr{E}\left[\sup _{t_{0} \leq t \leq T}\left|Y^{k}(t)\right|^{2}\right],
\end{aligned}
$$

we have

$$
\begin{aligned}
& \mathscr{E}\left[\sup _{t_{0} \leq u \leq t}\left|Y^{k}(u)\right|^{2}\right] \\
& \leq 4 \mathscr{E}\left[\|\xi\|^{2}\right]+4 \widetilde{K}\left(C_{1} T+C_{2} T+C_{3}\right) T \\
&+4 \widetilde{K}\left(C_{1} T+C_{2} T+C_{3}\right) \\
& \times \int_{t_{0}}^{t}\left(\mathscr{E}\left[\|\xi\|^{2}\right]+\mathscr{E}\left[\sup _{t_{0} \leq u \leq v}\left|Y^{k}(u)\right|^{2}\right]\right) d v \\
& \leq 4 \mathscr{E}\left[\|\xi\|^{2}\right]+4 \widetilde{K}\left(C_{1} T+C_{2} T+C_{3}\right) T \\
&+4 \widetilde{K} T\left(C_{1} T+C_{2} T+C_{3}\right) \mathscr{E}\left[\|\xi\|^{2}\right] \\
&+4 \widetilde{K}\left(C_{1} T+C_{2} T+C_{3}\right) \\
& \times \int_{t_{0}}^{t}\left(\widetilde{\mathscr{E}}\left[\sup _{t_{0} \leq u \leq v}\left|Y^{k}(u)\right|^{2}\right]\right) d v \\
&= K_{1}+4 \widetilde{K} K_{2} \\
& \times \int_{t_{0}}^{t}\left(\mathscr{E}\left[\sup _{t_{0} \leq u \leq v}\left|Y^{k}(u)\right|^{2}\right]\right) d v,
\end{aligned}
$$

where $K_{1}=4\left(1+T \widetilde{K} K_{2}\right) \mathscr{E}\left[\|\xi\|^{2}\right]+4 \widetilde{K} K_{2} T$ and $K_{2}=C_{1} T+$ $\mathrm{C}_{2} \mathrm{~T}+\mathrm{C}_{3}$. Thus the Gronwall inequality gives

$$
\mathscr{E}\left[\sup _{t_{0} \leq u \leq t}\left|Y^{k}(u)\right|^{2}\right] \leq K_{1} e^{4 \widetilde{K} K_{2} t}
$$

Letting $t=T$, we have

$$
\mathscr{E}\left[\sup _{t_{0} \leq u \leq T}\left|Y^{k}(u)\right|^{2}\right] \leq K_{1} e^{4 \widetilde{K} K_{2} T} .
$$


Consequently,

$$
\begin{aligned}
& \mathscr{E}\left[\sup _{t_{0}-\tau \leq u \leq T}\left|Y^{k}(u)\right|^{2}\right] \\
& \leq \mathscr{E}\left[\|\xi\|^{2}\right]+\mathscr{E}\left[\sup _{t_{0} \leq u \leq T}\left|Y^{k}(u)\right|^{2}\right] \\
& \leq \mathscr{E}\left[\|\xi\|^{2}\right]+K_{1} e^{4 \widetilde{K} K_{2} T}=C,
\end{aligned}
$$

which is the required result (22).

Remark 11. The above lemma shows that, for all $k \geq 1, Y^{k}(t) \in$ $M_{G}^{2}\left(\left[t_{0}-\tau, T\right] ; \mathbb{R}^{d}\right)$. Similarly, as the previous lemma, one can show that

$$
\sup _{t_{0}-\tau \leq t \leq T} \mathscr{E}\left[|Y(t)|^{2}\right] \leq C, \quad \text { for any } T>0
$$

Lemma 12. For all $k \geq 1$ and $t_{0} \leq s<t \leq T$,

$$
\mathscr{E}\left[\left|Y^{k}(t)-Y^{k}(s)\right|^{2}\right] \leq K_{3}(t-s),
$$

where $K_{3}=3 \widetilde{K} K_{2}\left(1+\mathscr{E}\|\xi\|^{2}+K^{*}\right)$ and $K^{*}=K_{1} e^{4 \widetilde{K} K_{2} T}$.

Proof. Obviously, one can observe that, for $k \geq 1$ and $t_{0} \leq s<$ $t \leq T$,

$$
\begin{aligned}
Y^{k}(t) & -Y^{k}(s) \\
= & \int_{s}^{t} \alpha\left(v, \widetilde{Y}^{k}(v)\right) d v \\
& +\int_{s}^{t} \beta\left(v, \widetilde{Y}^{k}(v)\right) d\langle B, B\rangle(v) \\
& +\int_{s}^{t} \gamma\left(v, \widetilde{Y}^{k}(v)\right) d B(v) .
\end{aligned}
$$

Thus using the linear growth condition (20) and the BDG inequalities [9] and taking sublinear expectation, we obtain

$$
\begin{aligned}
& \mathscr{E}\left[\sup _{s \leq r<u \leq t}\left|Y^{k}(u)-Y^{k}(r)\right|^{2}\right] \\
& \leq 3 \mathscr{E}\left[\sup _{s \leq r<u \leq t}\left|\int_{r}^{u} \alpha\left(v, \widetilde{Y}^{k}(v)\right) d v\right|^{2}\right] \\
& +3 \mathscr{E}\left[\sup _{s \leq r<u \leq t}\left|\int_{r}^{u} \beta\left(v, \widetilde{Y}^{k}(v)\right) d\langle B, B\rangle(v)\right|^{2}\right] \\
& +3 \mathscr{E}\left[\sup _{s \leq r<u \leq t}\left|\int_{r}^{u} \gamma\left(v, \widetilde{Y}^{k}(v)\right) d B(v)\right|^{2}\right]
\end{aligned}
$$

$$
\begin{aligned}
& \leq 3 C_{1}(t-s) \int_{s}^{t} \mathscr{E}\left[\left|\alpha\left(v, \widetilde{Y}^{k}(v)\right)\right|^{2}\right] d v \\
& +3 C_{2}(t-s) \int_{s}^{t} \mathscr{E}\left[\left|\beta\left(v, \widetilde{Y}^{k}(v)\right)\right|^{2}\right] d v \\
& +3 C_{3} \int_{s}^{t} \mathscr{E}\left[\left|\gamma\left(v, \widetilde{Y}^{k}(v)\right)\right|^{2}\right] d v \\
& \leq 3 \widetilde{K}\left(C_{1}(t-s)+C_{2}(t-s)+C_{3}\right) \\
& \times \int_{s}^{t}\left(1+\mathscr{E}\left[\left|\widetilde{Y}^{k}(v)\right|^{2}\right]\right) d v \\
& \leq 3 \widetilde{K}\left(C_{1} T+C_{2} T+C_{3}\right) \\
& \times \int_{s}^{t}\left(1+\mathscr{E}\left[\left|\tilde{Y}^{k}(v)\right|^{2}\right]\right) d v \\
& =3 \widetilde{K} K_{2}(t-s)+3 \widetilde{K} K_{2} \\
& \times \int_{s}^{t} \mathscr{E}\left[\left|\sup _{t_{0}-\tau \leq u \leq v} Y^{k}(u)\right|^{2}\right] d v \\
& \leq 3 \widetilde{K} K_{2}(t-s)+3 \widetilde{K} K_{2} \\
& \times \int_{s}^{t}\left(\mathscr{E}\left[\|\xi\|^{2}\right]+\mathscr{E}\left[\sup _{t_{0} \leq u \leq v}\left|Y^{k}(u)\right|^{2}\right]\right) d u \\
& \leq 3 \widetilde{K} K_{2}(t-s) \\
& +3 \widetilde{K} K_{2} \mathscr{E}\left[\|\xi\|^{2}\right](t-s) \\
& +3 \widetilde{K} K_{2} \int_{s}^{t} \mathscr{E}\left[\sup _{t_{0} \leq u \leq v}\left|Y^{k}(u)\right|^{2}\right] d u .
\end{aligned}
$$

From (29) taking $K^{*}=K_{1} e^{4 \widetilde{K} K_{2} T}$ we get

$$
\begin{aligned}
\mathscr{E} & {\left[\left|Y^{k}(t)-Y^{k}(s)\right|^{2}\right] } \\
\leq & 3 \widetilde{K} K_{2}(t-s)+3 \widetilde{K} K_{2} \mathscr{E}\left[\|\xi\|^{2}\right] \\
& \times(t-s)+3 \widetilde{K} K_{2} K^{*}(t-s) \\
\leq & 3 \widetilde{K} K_{2}\left[1+\mathscr{E}\|\xi\|^{2}+K^{*}\right](t-s) \\
= & K_{3}(t-s),
\end{aligned}
$$

which is the required result (32).

Remark 13. In a similar way as the previous lemma one can prove

$$
\mathscr{E}\left[|Y(t)-Y(s)|^{2}\right] \leq K_{3}(t-s) .
$$




\section{Existence of Solutions for G-SFDEs}

Firstly, this section shows that the unique solution of GSFDEs (15) gets convergence from Cauchy-Maruyama (CM) approximate solutions and then it determines that the GSFDE (15) has a unique solution with the Cauchy-Maruyama approximation scheme. Suppose if there is a constant $\theta$ that is positive such as

$$
\mathscr{E}\left[\left|\xi\left(a_{2}\right)-\xi\left(a_{1}\right)\right|^{2}\right] \leq \theta\left(a_{2}-a_{1}\right) \quad \text { if }-\tau \leq a_{1}<a_{2} \leq 0
$$

that is, the initial data $\xi=\{\xi(a):-\tau \leq a \leq 0\}$ is uniformly Lipschitz $L^{2}$-continuous.

Theorem 14. Suppose that the coefficients $\alpha, \beta$, and $\gamma$ satisfy the linear growth and Lipschitz conditions (20) and (21), respectively. Let the initial data satisfy condition (37). Then

$$
\mathscr{E}\left[\sup _{t_{0} \leq t \leq T}\left|Y^{k}(t)-Y(t)\right|^{2}\right] \leq \frac{C^{*}}{k},
$$

where $C^{*}=6 \widehat{K} K_{2}\left[C_{2} T+\left(C_{2} \vee \theta\right) \tau\right] e^{6 \widehat{K} K_{2} T}$.

Proof. Using the BDG inequalities [9], the Lipschitz condition (21), and inequality (32), we have

$$
\begin{aligned}
\mathscr{E}\left[\sup _{t_{0} \leq u \leq t}\left|Y^{k}(u)-Y(u)\right|^{2}\right] \\
\leq 3 \widehat{K} K_{2} \int_{t_{0}}^{t} \mathscr{E}\left[\left|\widetilde{Y}^{k}(v)-Y(v+a)\right|^{2}\right] d v \\
\leq 3 \widehat{K} K_{2} \int_{t_{0}}^{t} \mathscr{E}\left[\mid \widetilde{Y}^{k}(v)-Y(v+a)\right. \\
\leq 6 \widehat{K} K_{2} \int_{t_{0}}^{t} \mathscr{E}\left[\left|\widetilde{Y}^{k}(v)-\tilde{Y}(v)\right|^{2}\right] d v \\
+6 \widehat{K} K_{2} \int_{t_{0}}^{t} \mathscr{E}\left[|\tilde{Y}(v)-Y(v+a)|^{2}\right] d v \\
=6 \widehat{K} K_{2} \int_{t_{0}}^{t} \mathscr{E}\left[\sup _{t_{0} \leq u \leq v}\left|Y^{k}(u)-Y(u)\right|^{2}\right] d v+I,
\end{aligned}
$$

where $I=6 \widehat{K} K_{2} \int_{t_{0}}^{t} \mathscr{E}\left[|\widetilde{Y}(v)-Y(v+a)|^{2}\right] d v$. Then by Gronwall's inequality

$$
\mathscr{E}\left[\sup _{t_{0} \leq u \leq t}\left|Y^{k}(u)-Y(u)\right|^{2}\right] \leq I e^{6 \widehat{K} K_{2} T}
$$

Using (36) we estimate $I$ as follows:

$$
\begin{aligned}
I= & 6 \widehat{K} K_{2} \\
& \times \sum_{k \geq 0} \int_{t_{0}+(n / k)}^{\left(t_{0}+((n+1) / k)\right) \wedge T} \mathscr{E}\left[\mid Y\left(t_{0}+\frac{n}{k}+a\right)\right. \\
& \left.-\left.Y(v+a)\right|^{2}\right] d v \\
\leq & 6 \widehat{K} K_{2} C_{2}\left(T-t_{0}\right) \frac{1}{k}+6 \widehat{K} K_{2} \\
& \times \sum_{k \geq 0} \int_{t_{0}+(n / k)}^{\left(t_{0}+((n+1) / k)\right) \wedge \tau} \mathscr{E}\left[\mid Y\left(t_{0}+\frac{n}{k}+a\right)\right. \\
\leq & 6 \widehat{K} K_{2} C_{2} T \frac{1}{k}+6 \widehat{K} K_{2}\left(C_{2} \vee \theta\right) \tau \frac{1}{k} \\
= & 6 \widehat{K} K_{2}\left[C_{2} T+\left(C_{2} \vee \theta\right) \tau\right] \frac{1}{k} .
\end{aligned}
$$

Thus substituting the value of $I$ in (40), we obtain

$$
\begin{aligned}
& \mathscr{E}\left[\sup _{t_{0} \leq u \leq t}\left|Y^{k}(u)-Y(u)\right|^{2}\right] \\
& \quad \leq 6 \widehat{K} K_{2}\left[C_{2} T+\left(C_{2} \vee \theta\right) \tau\right] \frac{1}{k} e^{6 \widehat{K} K_{2} T}=\frac{C^{*}}{k},
\end{aligned}
$$

where $C^{*}=6 \widehat{K} K_{2}\left[C_{2} T+\left(C_{2} \vee \theta\right) \tau\right] e^{6 \widehat{K} K_{2} T}$, which is the required result (38).

Theorem 15. Suppose that the coefficients $\alpha, \beta$, and $\gamma$ satisfy the linear growth and Lipschitz conditions (20) and (21), respectively. Let the initial data satisfy condition (37). Then $\left\{Y^{k}, k \geq 1\right\}$ defined by (18) is a Cauchy sequence in $M_{G}^{2}\left(t_{0}-\right.$ $\left.\tau, T ; \mathbb{R}^{n}\right)$ and it converges to the unique solution $Y(t)$ of the $G$ SFDE (15).

Proof. Firstly, it is proved that the sequence $\left\{Y^{k}, k \geq 1\right\}$ is Cauchy. Assume $l>k$; then for $t \in\left[t_{0}, T\right]$,

$$
\begin{aligned}
& \left|Y^{l}(t)-Y^{k}(t)\right|^{2} \\
& \leq 3\left|\int_{t_{0}}^{t}\left[\alpha\left(v, \widetilde{Y}^{l}(v)\right)-\alpha\left(v, \tilde{Y}^{k}(v)\right)\right] d v\right|^{2} \\
& \quad+3\left|\int_{t_{0}}^{t}\left[\beta\left(v, \tilde{Y}^{l}(v)\right)-\beta\left(v, \tilde{Y}^{k}(v)\right)\right] d\langle B, B\rangle(v)\right|^{2} \\
& \quad+3\left|\int_{t_{0}}^{t}\left[\gamma\left(v, \tilde{Y}^{l}(v)\right)-\gamma\left(v, \tilde{Y}^{k}(v)\right)\right] d B(v)\right|^{2} .
\end{aligned}
$$


By using the BDG inequalities [9], Lipschitz condition (21), and Lemma (10), we get

$$
\begin{aligned}
\sup _{t_{0} \leq t \leq T} \mathscr{E} & {\left[\left|Y^{l}(t)-Y^{m}(t)\right|^{2}\right] } \\
\leq & 3 \widehat{K}\left(C_{1} T+C_{2} T+C_{3}\right) \\
& \times \int_{t_{0}}^{t} \mathscr{E}\left[\left|\widetilde{Y}^{l}(v)-\widetilde{Y}^{k}(v)\right|^{2}\right] d v \\
\leq & 6 \widehat{K} K_{2} \int_{t_{0}}^{t} \mathscr{E}\left[\left|\widetilde{Y}^{l}(v)-\widetilde{Y}(v)\right|^{2}\right] d v \\
& +6 \widehat{K} K_{2} \int_{t_{0}}^{t} \mathscr{E}\left[|\widetilde{Y}(v)|^{2}-\widetilde{Y}^{k}(v)\right] d v \\
\leq & 6 \widehat{K} K_{2} \int_{t_{0}}^{t} \sup _{t_{0} \leq u \leq v} \mathscr{E}\left[\left|Y^{l}(u)-Y(u)\right|^{2}\right] d v \\
& +6 \widehat{K} K_{2} \int_{t_{0}}^{t} \sup _{t_{0} \leq u \leq v} \mathscr{E}\left[\left|Y^{k}(u)-Y(u)\right|^{2}\right] d v .
\end{aligned}
$$

Using (38), it yields

$$
\begin{aligned}
& \sup _{t_{0} \leq t \leq T} \mathscr{E}\left[\left|Y^{l}(t)-Y^{k}(t)\right|^{2}\right] \\
& \leq 6 \widehat{K} K_{2} \int_{t_{0}}^{t} \frac{C^{*}}{l} d v+6 \widehat{K} K_{2} \int_{t_{0}}^{t} \frac{C^{*}}{k} d v \\
& \leq 6 \widehat{K} K_{2} T C^{*}\left(\frac{1}{l}+\frac{1}{k}\right) .
\end{aligned}
$$

Consequently, we have

$$
\begin{aligned}
& \sup _{t_{0} \leq t \leq T} \mathscr{E}\left[\left|Y^{l}(t)-Y^{k}(t)\right|^{2}\right] \\
& \quad \leq C_{1}^{*}\left(\frac{1}{k}+\frac{1}{l}\right) \longrightarrow 0, \quad \text { when } k, l \longrightarrow \infty
\end{aligned}
$$

where $C_{1}^{*}=6 \widehat{K} K_{2} T C^{*}$. Thus the sequence $\left\{X^{k}(t), k \geq 1\right\}$ is a Cauchy sequence in $M_{G}^{2}\left(\left[t_{0}-\tau, T\right] ; \mathbb{R}^{n}\right)$. Denoting its limits by $X(t)$ and letting $k \rightarrow \infty$ in (46) yield

$$
\sup _{t_{0}-\tau \leq t \leq T} \mathscr{E}\left[\left|Y^{l}(t)-Y(t)\right|^{2}\right] \leq \frac{C_{1}^{*}}{l} .
$$

Now we show that $Y(t)$ satisfies the G-SFDE (15). Let $t \in$ $\left[t_{0}, T\right]$; then using the Lipschitz condition (21), Lemma 10, and the BDG inequalities [9], we get

$$
\begin{aligned}
\mathscr{E}\left[\left|\int_{t_{0}}^{t}\left[\alpha\left(v, \widetilde{Y}^{k}(v)\right)-\alpha(v, Y(v+a))\right] d v\right|^{2}\right] \\
+\mathscr{E}\left[\mid \int_{t_{0}}^{t}\left[\beta\left(v, \widetilde{Y}^{k}(v)\right)\right.\right. \\
\left.-\beta(v, Y(v+a))]\left.d\langle B, B\rangle(v)\right|^{2}\right] \\
+\mathscr{E}\left[\mid \int_{t_{0}}^{t}\left[\gamma\left(v, \widetilde{X}^{k}(v)\right)\right.\right. \\
\left.\quad-\gamma(v, X(v+a))]\left.d B(v)\right|^{2}\right] \\
\leq \widehat{K} K_{2} \int_{t_{0}}^{t} \mathscr{E}\left[\left|\widetilde{Y}^{k}(v)-Y(v+a)\right|^{2}\right] d v \\
\leq \widehat{K} K_{2} \int_{t_{0}}^{t} \mathscr{E}\left[\left|\widetilde{Y}^{k}(v)-\widetilde{Y}(v)\right|^{2}\right] d v \\
+\widehat{K} K_{2} \int_{t_{0}}^{t} \mathscr{E}\left[|\widetilde{Y}(v)-Y(v+a)|^{2}\right] d v . \\
\leq \widehat{K} K_{2} \int_{t_{0}}^{t} \mathscr{E}\left[\sup _{t_{0} \leq u \leq v}\left|Y^{k}(u)-Y(u)\right|^{2}\right] d v+\frac{I}{6} . \\
\leq \widehat{K} K_{2} T \frac{C^{*}}{k}+\widehat{K} K_{2}\left[C_{2} T+\left(C_{2} \vee \theta\right) \tau\right] \frac{1}{k} \\
=\widehat{K} K_{2}\left[T C^{*}+C_{2} T+\left(C_{2} \vee \theta\right) \tau\right] \frac{1}{k} \\
\longrightarrow 0 \quad \operatorname{as} k \longrightarrow \infty,
\end{aligned}
$$

where $C_{1}^{*}=\widehat{K} K_{2}\left[T C^{*}+C_{2} T+\left(C_{2} \vee \theta\right) \tau\right]$. Thus we get $Y(t)$ as a solution of (15). To show the uniqueness, assume that the G-SFDE (15) has two solutions, say $Y(t)$ and $Z(t)$. Then in a similar fashion as above one can prove that

$$
\mathscr{E}\left[\sup _{t_{0} \leq t \leq T}|Z(t)-Y(t)|^{2}\right]=0
$$

which gives $Z(t)=Y(t)$ for $t \in\left[t_{0}, T\right]$. Hence for all $t \epsilon$ $\left[t_{0}-\tau, T\right], Z(t)=Y(t)$ q.s.

\section{Conflict of Interests}

The author declares that there is no conflict of interests regarding the publication of this paper. 


\section{Acknowledgments}

The author appreciates and acknowledges the financial support of NUST Pakistan. The author acknowledges the careful reading and some very useful suggestions of Dr. Ali Anwar.

\section{References}

[1] X. Mao, Stochastic Differential Equations and Their Applications, Horwood, Chichester, UK, 1997.

[2] R. Z. Has'minskii, Stochastic Stability of Differential Equations, Sijthoff \& Noordhoff, 1980.

[3] X. Mao, Exponential Stability of Stochastic Differential Equations, Marcel Dekker, New York, NY, USA, 1994.

[4] S. Peng, "G-expectation, G-Brownian motion and related stochastic calculus of It o type," in Proceeeding of the Abel Symposium, F. E. Benth, G. Di Nunno, T. Lindstrom, B. Ksendal, and T. Zhang, Eds., pp. 541-567, Springer, Berlin, Germany, 2006.

[5] S. Peng, "Multi-dimensional G-Brownian motion and related stochastic calculus under G-expectation," Stochastic Processes and their Applications, vol. 118, no. 12, pp. 2223-2253, 2008.

[6] X. Chen, "Dynkin's formula under the G-expectation," Statistics \& Probability Letters, vol. 80, no. 5-6, pp. 519-526, 2010.

[7] F. Faizullah, "A note on the caratheodory approximation scheme for stochastic differential equations under G-Brownian motion," Zeitschrift fur Naturforschung A: Journal of Physical Sciences, vol. 67, no. 12, pp. 699-704, 2012.

[8] F. Faizullah, W. A. Khan, M. Arif, and R. A. Khan, "On the existence of solutions for stochastic differential equations under G-Brownian motion," Life Science Journal, vol. 10, no. 5, pp. 255260, 2013.

[9] F. Gao, "Pathwise properties and homeomorphic flows for stochastic differential equations driven by $G$-Brownian motion," Stochastic Processes and Their Applications, vol. 119, no. 10, pp. 3356-3382, 2009.

[10] J. Xu and B. Zhang, "Martingale characterization of G-Brownian motion," Stochastic Processes and their Applications, vol. 119, no. 1, pp. 232-248, 2009.

[11] Y. Ren, Q. Bi, and R. Sakthivel, "Stochastic functional differential equations with infinite delay driven by $G$-Brownian motion," Mathematical Methods in the Applied Sciences, vol. 36, no. 13, pp. 1746-1759, 2013.

[12] L. Denis, M. Hu, and S. Peng, "Functional spaces and capacity related to a sulinear expectations: application to G-Brownian motion paths," Potential Analysis, vol. 34, no. 2, pp. 139-161, 2011.

[13] F. Faizullah, "Existence of solutions for stochastic differential equations under G-Brownian motion with discontinuous coefficients," Zeitschrift fur Naturforschung A, vol. 67, no. 12, pp. 692698, 2012. 


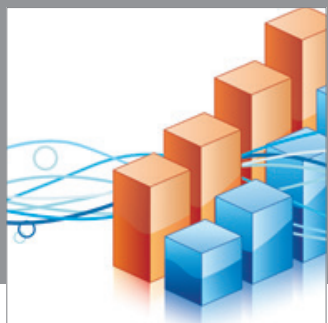

Advances in

Operations Research

mansans

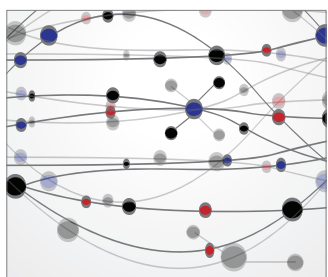

The Scientific World Journal
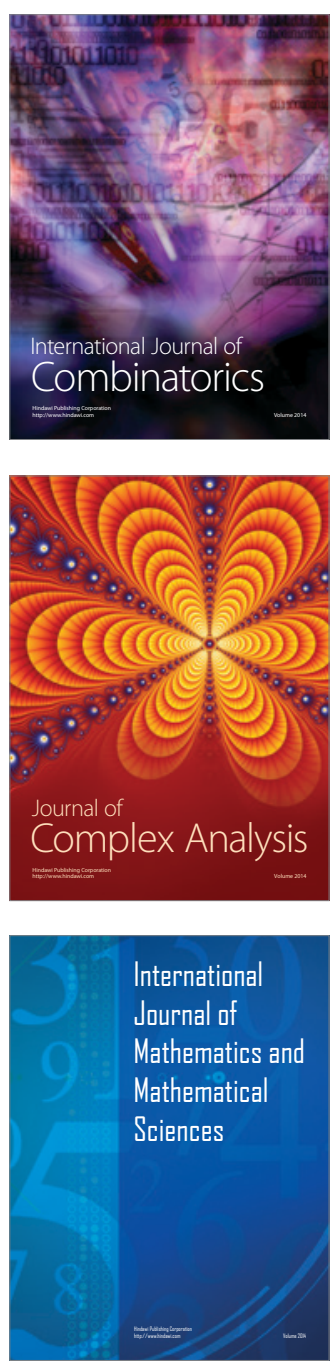
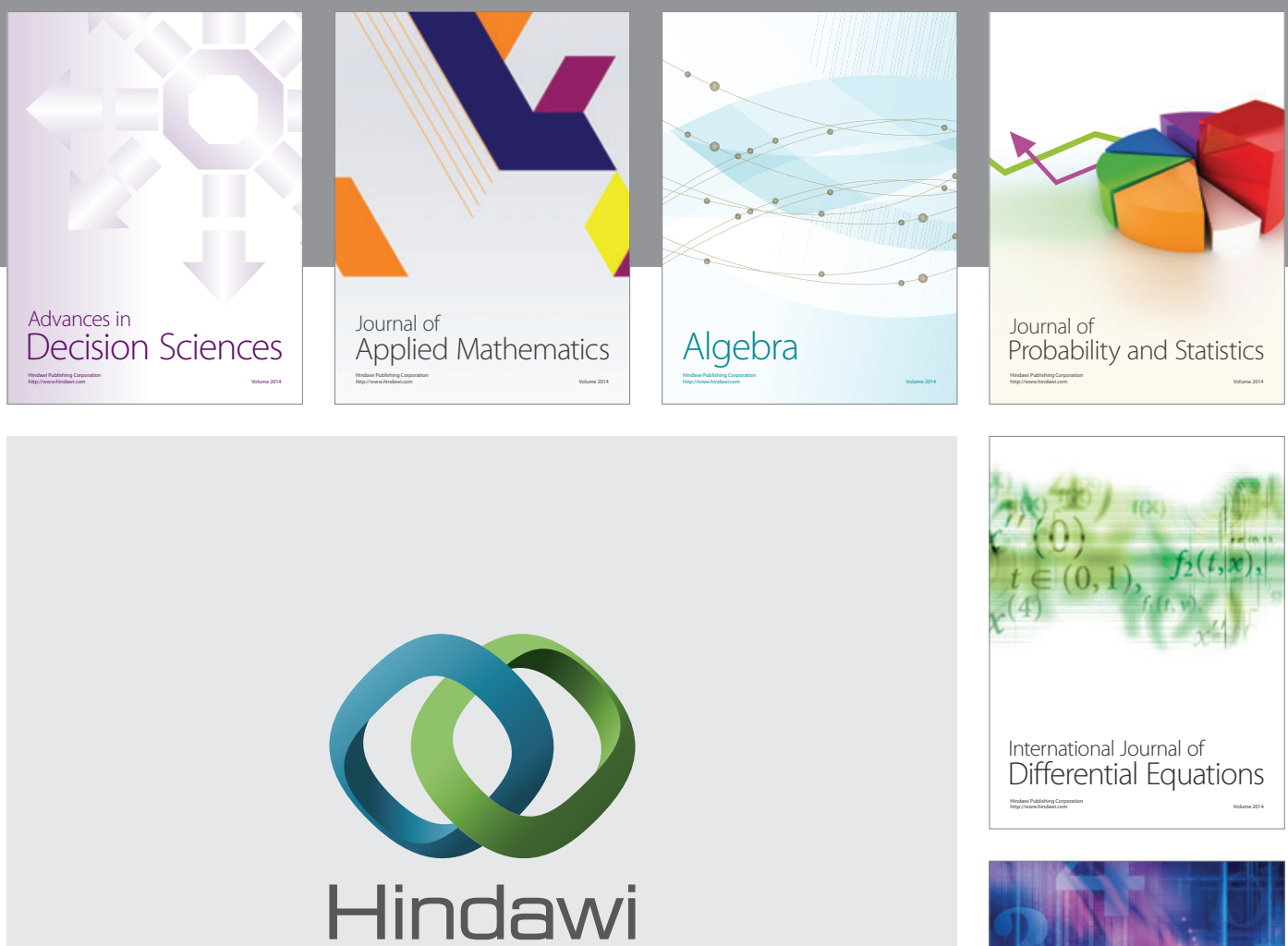

Submit your manuscripts at http://www.hindawi.com
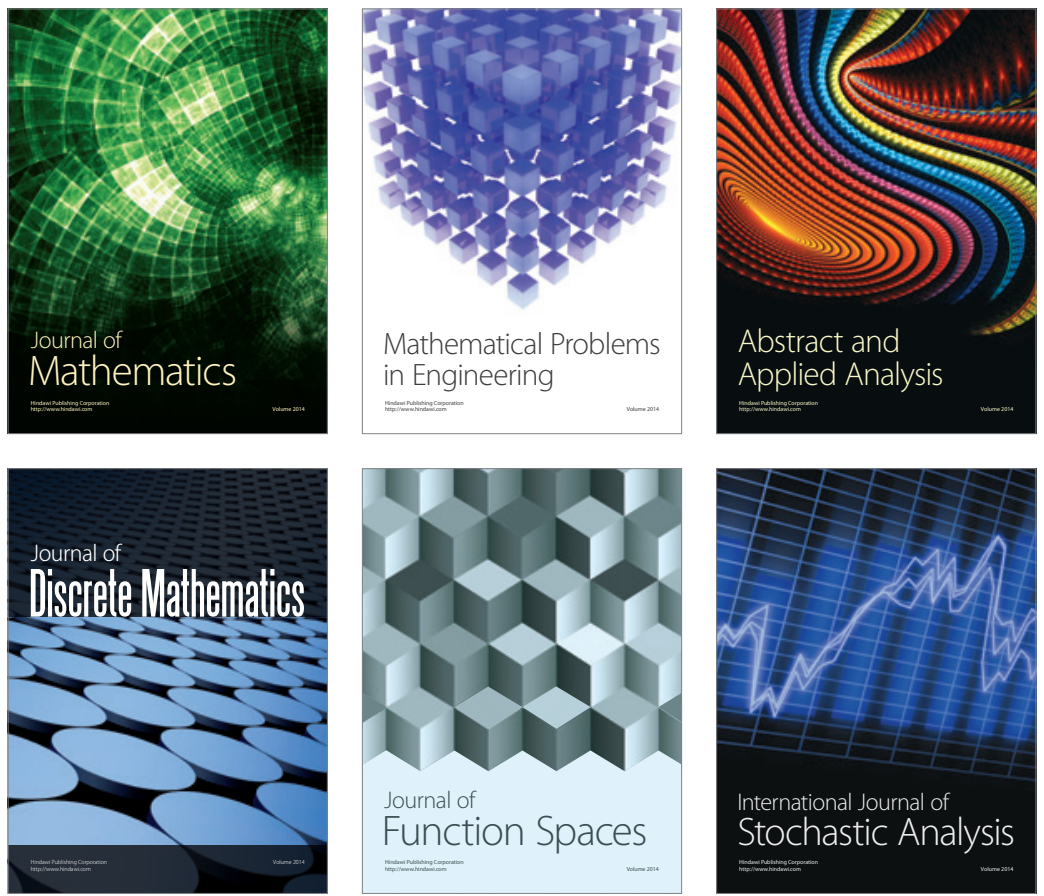

Journal of

Function Spaces

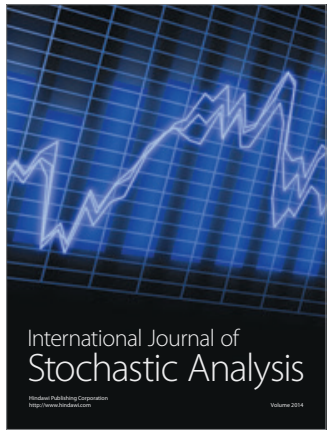

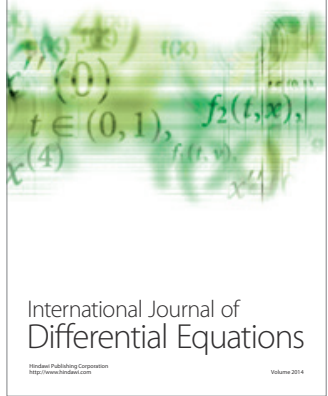
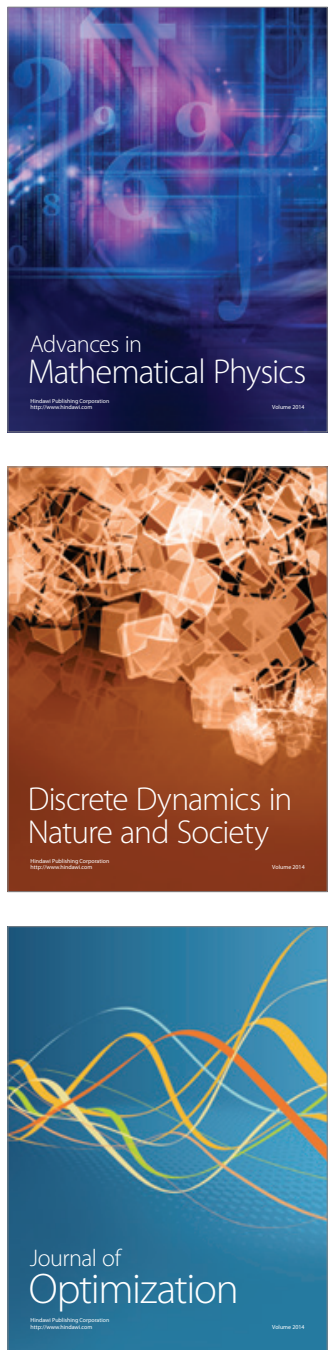